\title{
Solvable model for spatiotemporal chaos
}

\author{
R. O. Grigoriev and H. G. Schuster* \\ Condensed Matter Physics 114-36 and Neural Systems Program 139-74, California Institute of Technology, Pasadena, California 91125
}

(Received 6 June 1997; revised manuscript received 22 September 1997)

\begin{abstract}
We show that the dynamical behavior of a coupled map lattice where the individual maps are Bernoulli shift maps can be solved analytically for integer couplings. We calculate the invariant density of the system and show that it displays a nontrivial spatial behavior. We also introduce and calculate a generalized spatiotemporal correlation function. [S1063-651X(98)10001-6]

PACS number(s): 05.45.+b, 05.50.+q
\end{abstract}

\section{INTRODUCTION}

The study of temporal chaos in low-dimensional systems, some of which can be described by low-dimensional maps $[1,2]$, was extremely beneficial for the understanding of turbulence. In 1984 coupled map lattices (CMLs) were introduced into the physical literature as a tool for studying spatiotemporal chaos in spatially extended, i.e., highdimensional systems [3]. They consist of spatially coupled low-dimensional maps and represent dynamical systems that are discrete in space and time, but continuous in the state variable. They serve as models for coupled Josephson junctions, excitable media, population dynamics, neural dynamics, and turbulence [4].

Although a number of statements regarding the appearance of coherent structures from spatiotemporal chaos were proved analytically (see, for instance, the works by Bunimovich and Sinai [5]), most results in the field have been obtained by numerical simulations $[4,6]$. The study of temporal chaos has greatly profited from the existence of simple maps such as the Bernoulli shift map and the cat map [2,7], which can be solved explicitly (for integer expansion rates), thereby making the mechanisms of mixing and temporal chaos understandable.

Unfortunately, no in-depth investigation of this type has been provided up to now for the problem of spatiotemporal chaos and coherent structures. The biggest progress was achieved for the class of coupled maps [3]

$$
z_{i}^{t+1}=(1-\varepsilon) f\left(z_{i}^{t}\right)+\frac{\varepsilon}{2}\left[f\left(z_{i+1}^{t}\right)+f\left(z_{i-1}^{t}\right)\right],
$$

with piecewise-linear local maps $f\left(z_{i}^{t}\right)$.

In particular, Keller et al. [8] have shown for $f(x)=(2 x) \bmod 1$ that the dynamics of the CML (1) with $i$ $\in[1, N]$ is ergodic and the system possesses an invariant measure for sufficiently small coupling $\varepsilon$. However, for certain larger values of coupling the dynamics becomes nonergodic. Several specific results for $N=2$ were also obtained.

The case $f(x)=(a x) \bmod 1$ for arbitrary expansion rates $a$ was considered in the limit of an infinite lattice $i$ $\in(-\infty, \infty)$ by Diks et al. [9], who have calculated the den-

\footnotetext{
*Permanent address: Institute für Theoretische Physik, Universität Kiel, Olshausenstrasse 40, D 24098 Kiel, Germany.
}

sities of the information dimension and information entropy and also suggested that the evolution equation (1) could be solved for certain values of $a$ and $\varepsilon$ if rewritten in the equivalent form

$$
x_{i}^{t+1}=f\left((1-\varepsilon) x_{i}^{t}+\frac{\varepsilon}{2}\left(x_{i+1}^{t}+x_{i-1}^{t}\right)\right) \text {, }
$$

using the change of variables $x_{i}^{t}=f\left(z_{i}^{t}\right)$.

In the present paper we will actually solve a more general evolution equation, which gives the solution to the CML (2) as well as its versions for spatial coupling of arbitrary range, and calculate the invariant measure of respective systems. We will also obtain the conditions that determine when the evolution of the system is ergodic and when the ergodicity breaks down.

To make our approach for the high-dimensional system more transparent we will start by calculating in Sec. II the invariant density and a temporal correlation function for the single Bernoulli shift map. In Sec. III we will show that a wide class of finite-dimensional evolution equations, which includes the CML (2) as a special case for certain values of the coefficients $a$ and $\varepsilon$, can be solved and we will also calculate the invariant density for any such evolution equation. We will then use the general results to calculate the spatial and spatiotemporal correlation functions for the CML (2). Finally, in Sec. IV we will discuss our results and indicate directions of further research.

\section{PROPERTIES OF A SINGLE BERNOULLI SHIFT MAP}

First we recall that the single map

$$
x^{t+1}=\left(a x^{t}\right) \bmod 1
$$

can be solved as $x^{t}=\left(a^{t} x^{0}\right) \bmod 1$ because

$$
\begin{aligned}
x^{2} & =\left\{a\left[\left(a x^{0}\right) \bmod 1\right]\right\} \bmod 1=\left\{a\left[a x^{0}-k^{0}\right]\right\} \bmod 1 \\
& =\left(a^{2} x^{0}\right) \bmod 1,
\end{aligned}
$$

where $k^{0}$ is an integer that represents the action of the modulo function. For the last equality sign in Eq. (4) to hold we require $a$ to be an integer such that $a k^{0}$ becomes again an integer, which can be dropped within the last modulo function. 
Since the modulo function confines the variable $x^{t}$ to a circle, we could view the Bernoulli shift map as a linear map $x^{t+1}=a x^{t}$ where the variables exist on a unit circle, i.e., on a 1-torus. Similarly, we shall see below that we can view our coupled map system as a linear map acting on variables confined to an $N$-torus, where $N$ is the number of lattice sites.

The invariant density $\rho(x)$ of the simple Bernoulli shift map measures the distribution of $x$ values on the attractor generated by the map and is well known to be a constant [2] (here and below in this section we assume $|a|>1$ ). We can obtain this result by noting that $\rho(x)$ is defined on a unit circle, i.e., it is periodic in $x$ and therefore can be represented as a Fourier series

$$
\rho(x)=\sum_{k} \hat{\rho}(k) e^{2 \pi i k x},
$$

where $k$ takes only integer values $k=0, \pm 1, \pm 2, \ldots$.

The density $\rho^{t}(x)$ of the points on the attractor evolves from an initial distribution $\rho^{0}(x)$ according to the FrobeniusPerron equation [2]

$$
\rho^{t}(x)=\int_{0}^{1} d x^{\prime} \delta\left(x-\left(a^{t} x^{\prime}\right) \bmod 1\right) \rho^{0}\left(x^{\prime}\right),
$$

and since the map (3) is mixing, the invariant density is given by the long-time limit $\rho(x)=\lim _{t \rightarrow \infty} \rho^{t}(x)$.

In order to solve Eq. (6) we use Eq. (5) and the fact that the Bernoulli shift map becomes a linear map on a torus, such that

$$
\exp \left\{2 \pi i\left[\left(a^{t} x\right) \bmod 1\right]\right\}=\exp \left(2 \pi i a^{t} x\right)
$$

to obtain

$$
\hat{\rho}^{t}(k)=\hat{\rho}^{0}\left(a^{t} k\right) .
$$

If we make the reasonable assumption that the initial distribution $\rho^{0}(x)$ is nonsingular, then $\lim _{k \rightarrow \pm \infty} \hat{\rho}^{0}(k)=0$. This means that all Fourier coefficients $\hat{\rho}^{t}(k)$ in Eq. (5) tend to zero in the infinite-time limit, except the one that belongs to $k=0$. Since

$$
\hat{\rho}^{0}(0)=\int_{0}^{1} d x \rho^{0}(x)=1,
$$

this yields $\hat{\rho}(k)=\delta_{k, 0}$ and $\rho(x)=1$.

In a similar fashion we can now define and calculate the time correlation function on the 1-torus. Due to the nontrivial topology of the state space the usual time correlation function

$$
\left\langle x^{0} x^{t}\right\rangle=\int_{0}^{1} d x \rho(x) x f^{t}(x),
$$

where the time evolution of $x$ is given by the map $f(x)$, might produce confusing results. It is therefore advantageous to introduce the time correlation function

$$
G(t)=\int_{0}^{1} d x^{0} \rho\left(x^{0}\right) e^{2 \pi i\left(x^{0}-x^{t}\right)},
$$

where

$$
x^{t}=\left(a^{t} x^{0}\right) \bmod 1,
$$

which respects the fact that the variable $x$ is an angular variable on a torus [10]. Since $\rho(x)=1$ for $|a|>1$, Eqs. (7) and (11) yield

$$
G(t)=\int_{0}^{1} d x \rho(x) e^{2 \pi i\left(1-a^{t}\right) x}=\delta_{t, 0},
$$

i.e., the correlation vanishes for finite times.

In the following section we will demonstrate what changes have to be made in order to compute in a fashion similar to that above the solution to the dynamical equations, the invariant density, and the time correlation function for our coupled map lattice.

\section{LATTICE OF COUPLED BERNOULLI SHIFT MAPS}

\section{A. Evolution equation}

For the Bernoulli shift map $f(x)=(a x) \bmod 1$ the time evolution for the variables $x_{i}^{t}$ of the CML becomes, according to Eq. (2),

$$
x_{i}^{t+1}=\left(a\left[(1-\varepsilon) x_{i}^{t}+\frac{\varepsilon}{2}\left(x_{i+1}^{t}+x_{i-1}^{t}\right)\right]\right) \bmod 1 .
$$

If we introduce two new parameters $m$ and $n$, such that $a=m+2 n$ and $\varepsilon=2 n /(m+2 n)$, then the equation of motion for the coupled map system can be written in the compact form

$$
x_{i}^{t+1}=\left(\sum_{j} A_{i j} x_{j}^{t}\right) \bmod 1,
$$

where the coupling matrix $A$ has elements

$$
A_{i j}=m \delta_{i, j}+n\left(\delta_{i, i+1}+\delta_{i, i-1}\right) .
$$

This model was independently considered in the limit of an infinitely large lattice by Diks et al. [9], who recognized that the evolution equation (15) can be solved analytically for arbitrary integer parameters $m$ and $n$ [such that both $(1-\varepsilon) a$ and $a \varepsilon / 2$ take integer values] and used this fact to show that the components of the state $x_{i}^{t}$ represent an ensemble of independent identically distributed random variables with the uniform probability density on $[0,1)$. This is true, however, only for certain combinations of $m$ and $n$. In general, the variables $x_{i}^{t}$ are not independent, i.e., there is a nontrivial coherent structure, although more sophisticated methods are necessary to show this by calculating the natural invariant measure $\mu(\mathbf{x})$, which defines the invariant density $\rho(\mathbf{x})$ of the system through $d \mu(\mathbf{x})=\rho(\mathbf{x}) d \mathbf{x}$ [11]. In particular we will find that in certain cases the system might not be ergodic, and even if it is, the invariant measure might be nonconstant.

Below we present the solution of a more general problem of calculating an invariant measure of the system (15) of arbitrary length $N$ for a wide class of matrices $A$. Indeed, we can free ourselves from the specific form (16) for $A_{i j}$, which was physically motivated by the nearest-neighbor lattice 
model (14) and show that Eq. (15) can be solved for any matrix $A$ that has integer valued elements $A_{i j}$. In order to see this we write Eq. (15) in vector notation as

$$
\mathbf{x}^{t+1}=\left(A \mathbf{x}^{t}\right) \bmod 1
$$

where $\mathbf{x}^{t}=\left(x_{1}^{t}, \ldots, x_{N}^{t}\right)$ and the modulo is taken for each component of the vector $A \mathbf{x}^{t}$. Then we obtain by iterating from the initial condition

$$
\mathbf{x}^{t+1}=\left(A \mathbf{x}^{t}\right) \bmod 1=A \mathbf{x}^{t}-\mathbf{k}^{t},
$$

where $\mathbf{k}^{t}$ is a vector with integer components, which represents the action of the modulo function. This yields

$$
\begin{aligned}
\mathbf{x}^{t+2} & =\left(A \mathbf{x}^{t+1}\right) \bmod 1=\left(A\left[A \mathbf{x}^{t}-\mathbf{k}^{t}\right]\right) \bmod 1 \\
& =\left(A A \mathbf{x}^{t}-A \mathbf{k}^{t}\right) \bmod 1=\left(A^{2} \mathbf{x}^{t}\right) \bmod 1,
\end{aligned}
$$

where the last equality sign holds only because all elements of the matrix $A$ are integers, such that $A \mathbf{k}^{t}$ is a vector with integer components, which can be dropped under the last modulo function. Since Eq. (19) holds for any $t$, we obtain the closed-form solution as a function of the initial value

$$
\mathbf{x}^{t}=\left(A^{t} \mathbf{x}^{0}\right) \bmod 1 .
$$

Equation (19) shows that we can solve not only the coupled map lattice problem (14), but all linearly coupled systems where the coupling occurs via a matrix $A$ with integer elements $A_{i j}$ and the nonlinearity is provided by the modulo function. The solution can be obtained by first solving the linear problem, i.e., by obtaining $A^{t} \mathbf{x}^{0}$ and then taking the modulo, which is the same as having the linear map acting on an $N$-torus in analogy to the famous Arnold cat map in two dimensions [7].

Next we investigate the invariant density and the spatiotemporal correlation function of the CML. The first quantity gives us information about the measurable time-averaged spatial structures in the system and the second one tells us about the measurable spatiotemporal structures.

\section{B. Invariant density}

Similarly to the one-dimensional case, the invariant density $\rho(\mathbf{x})$ yields the distribution of points on the attractor generated by the map $\mathbf{x}^{t+1}=\left(A \mathbf{x}^{t}\right) \bmod 1$. Assuming that the map is mixing, it could be obtained by starting from an initial distribution $\rho^{0}(\mathbf{x})$ as the infinite-time limit of $\rho^{t}(\mathbf{x})$ in the Frobenius-Perron equation

$$
\rho^{t}(\mathbf{x})=\int d \mathbf{x}^{\prime} \delta\left(\mathbf{x}-\left(A^{t} \mathbf{x}^{\prime}\right) \bmod 1\right) \rho^{0}\left(\mathbf{x}^{\prime}\right) .
$$

Although the mixing property might not hold for every matrix $A$ with integer elements, we expect the violation of this property to be an exception rather than the rule. In particular it was proved [8] that the map (14) possesses an invariant mixing measure for

$$
|a|>2, \quad \varepsilon<\bar{\varepsilon}
$$

where $0<\bar{\varepsilon}<1$ is some constant (it was suggested that this result will in fact hold for all $|a|>1$ ).
Since all quantities involved in Eq. (21) are periodic on an $N$-torus, the Fourier decomposition of $\rho^{t}(\mathbf{x})$ contains only wave vectors $\mathbf{k}$ with integer components $\left(\mathbf{k} \in Z^{N}\right)$ :

$$
\rho^{t}(\mathbf{x})=\sum_{\mathbf{k}} \hat{\rho}^{t}(\mathbf{k}) e^{2 \pi i \mathbf{k} \cdot \mathbf{x}}
$$

By using the $N$-dimensional generalization of the equality (7)

$$
\exp \left\{2 \pi i \mathbf{k} \cdot\left[\left(A^{t} \mathbf{x}\right) \bmod 1\right]\right\}=\exp \left\{2 \pi i\left[\left(A^{t}\right)^{T} \mathbf{k}\right] \cdot \mathbf{x}\right\}
$$

one obtains from Eqs. (21) and (23)

$$
\hat{\rho}^{t}(\mathbf{k})=\hat{\rho}^{0}\left(\left(A^{t}\right)^{T} \mathbf{k}\right) .
$$

If the initial distribution $\rho^{0}(\mathbf{x})$ is nonsingular, all Fourier coefficients vanish for large values of the wave vector:

$$
\lim _{|\mathbf{k}| \rightarrow \infty} \hat{\rho}^{0}(\mathbf{k})=0
$$

For a completely expanding map, where all eigenvalues of the matrix $A$ have an absolute value larger than one, $\lim _{t \rightarrow \infty}\left(A^{t}\right)^{T} \mathbf{k}=\infty$ for each $\mathbf{k} \neq \mathbf{0}$ and the only nonvanishing Fourier component becomes

$$
\hat{\rho}^{0}(\mathbf{0})=\int d \mathbf{x} \rho^{0}(\mathbf{x})=1
$$

which yields a constant invariant density

$$
\rho(\mathbf{x})=1
$$

This result is completely analogous to the single map case. However, we may obtain different results for the invariant density if there are nonexpanding directions in the phase space.

Indexing the stable $(|\lambda|<1)$, central $(|\lambda|=1)$, and unstable $(|\lambda|>1)$ eigenvalues and right (left) eigenvectors $\mathbf{e}$ ( $\widetilde{\mathbf{e}})$ of the matrix $A^{T}$ with indices $s, c$, and $u$, respectively, we have

$$
\left(A^{t}\right)^{T} \mathbf{k}=\sum_{p=s, c, u} \lambda_{p}^{t}\left(\widetilde{\mathbf{e}}^{p} \cdot \mathbf{k}\right) \mathbf{e}^{p}
$$

According to the above, we will obtain results that differ from the trivial expanding case only if there exists at least one $\mathbf{k} \neq \mathbf{0}$ such that its components along the unstable directions are all zero, i.e., it is contained in the direct sum of the stable and central manifolds $\left(\mathbf{k} \in W^{c} \oplus W^{s}\right)$ of the fixed point $\mathbf{k}=\mathbf{0}$ of the "conjugate" map

$$
\mathbf{k}^{t+1}=A^{T} \mathbf{k}^{t} .
$$

On the other hand, Eqs. (25) and (29) tell us that it is not enough to have central or contracting eigenvalues in order to get a nonconstant invariant density.

Let us first consider the case with a single stable direction $\mathbf{e}^{s}$ and no central directions. Since all components $k_{i}$ of a vector $\mathbf{k}$ are integers, it is contained within the stable mani- 


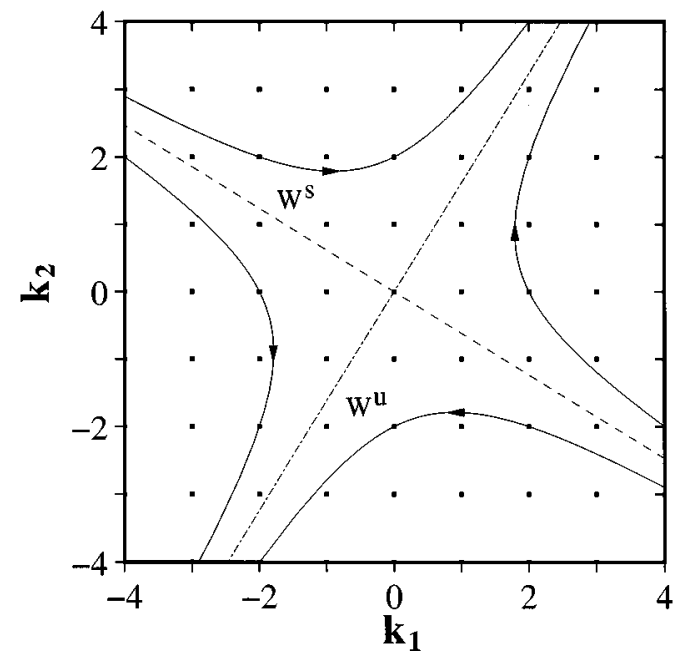

FIG. 1. Cat map: None of the integer-component wave vectors lies on the stable manifold $W^{s}$ of the fixed point $\mathbf{k}=\mathbf{0}$.

fold $W^{s}$ only if there exists a scalar $\nu$ such that $\nu \mathbf{k}=\mathbf{e}^{s}$. This in turn means that the components $\left\{e_{1}^{s}, \ldots, e_{N}^{s}\right\}$ should be mutually rational, i.e.,

$$
e_{1}^{s}: e_{2}^{s}: \cdots: e_{N}^{s}=k_{1}: k_{2}: \cdots: k_{N}
$$

An example where we have one contracting and one expanding direction is the cat map

$$
A=\left(\begin{array}{ll}
1 & 1 \\
1 & 2
\end{array}\right)
$$

Although the eigenvalue corresponding to the contracting direction is $\lambda_{s}=(3-\sqrt{5}) / 2<1$, this map still has a constant invariant density because the components of the eigenvector $\mathbf{e}^{s}=(2,1-\sqrt{5})$ belonging to $\lambda_{s}$ have a nonrational ratio (see Fig. 1) leading to

$$
\lim _{t \rightarrow \infty} \hat{\rho}^{t}(\mathbf{k})=\delta_{\mathbf{k}, 0}
$$

Generally, in order to get a nonconstant invariant density our model must possess a central and/or a stable manifold, i.e., $W^{c} \oplus W^{s} \neq\{\mathbf{0}\}$, whose direct sum in turn should contain at least one vector with mutually rational components. Every vector $\mathbf{k} \in \mathbb{Z}^{N}$ such that $\mathbf{k} \in\left(W^{c} \oplus W^{s}\right) \backslash W^{c}$ is pulled in the long-time limit into the origin, according to Eq. (29):

$$
\lim _{t \rightarrow \infty} \hat{\rho}^{t}(\mathbf{k})=\lim _{t \rightarrow \infty} \hat{\rho}^{0}\left(\left(A^{t}\right)^{T} \mathbf{k}\right)=\hat{\rho}^{0}(\mathbf{0})=1
$$

Similarly, vectors $\mathbf{k} \in \mathbb{Z}^{N}$ such that $\mathbf{k} \in W^{c}$ are either left invariant under the action of the map (30), and then

$$
\hat{\rho}^{t}(\mathbf{k})=\hat{\rho}^{0}(\mathbf{k})=e^{-2 \pi i \mathbf{k} \cdot \mathbf{x}^{0}},
$$

or change sign after each iteration, giving

$$
\hat{\rho}^{t}(\mathbf{k})=\left\{\begin{array}{lll}
\hat{\rho}^{0}(\mathbf{k})=e^{-2 \pi i \mathbf{k} \cdot \mathbf{x}^{0}}, & t \text { even } \\
\hat{\rho}^{1}(\mathbf{k})=e^{-2 \pi i \mathbf{k} \cdot \mathbf{x}^{1}}, & t \text { odd } .
\end{array}\right.
$$

As a result, the definition of the invariant density has to be adjusted to read

$$
\begin{aligned}
\rho(\mathbf{x}) & =\frac{1}{2} \lim _{t \rightarrow \infty}\left[\rho^{t}(\mathbf{x})+\rho^{t+1}(\mathbf{x})\right] \\
& =\frac{1}{2} \sum_{\mathbf{k}} \lim _{t \rightarrow \infty}\left[\rho^{t}(\mathbf{k})+\rho^{t+1}(\mathbf{k})\right] e^{2 \pi i \mathbf{k} \cdot \mathbf{x}} .
\end{aligned}
$$

The invariant density will contain only nonvanishing Fourier components with wave vectors $\mathbf{k} \in W^{c} \oplus W^{s}$ :

$$
\begin{aligned}
\rho(\mathbf{x})= & \sum_{\mathbf{k} \in\left(W^{c} \oplus W^{s}\right) \backslash W^{c}} e^{2 \pi i \mathbf{k} \cdot \mathbf{x}}+\frac{1}{2} \sum_{\mathbf{k} \in W^{c}}\left(e^{2 \pi i \mathbf{k} \cdot\left(\mathbf{x}-\mathbf{x}^{0}\right)}\right. \\
& \left.+e^{2 \pi i \mathbf{k} \cdot\left(\mathbf{x}-\mathbf{x}^{1}\right)}\right) .
\end{aligned}
$$

Using the fact that any $\mathbf{k} \in \mathbb{Z}^{N} \cap\left(W^{c} \oplus W^{s}\right)$ can be represented as a linear combination of a (usually small) number of basis vectors $\mathbf{f}^{j}, j=1, \ldots, M$, with integer coefficients $p_{j}$, i.e., $\mathbf{k}=\Sigma_{j} p_{j} \mathbf{f}^{j}$, we can rewrite Eq. (38) as

$$
\begin{aligned}
\rho(\mathbf{x})= & \prod_{j=c, s} \sum_{p_{j}} e^{2 \pi i p_{j} \mathbf{f}^{j} \cdot \mathbf{x}_{-}} \prod_{j=c} \sum_{p_{j}} e^{2 \pi i p_{j} \mathbf{f}^{j} \cdot \mathbf{x}} \\
& +\frac{1}{2} \sum_{t=0}^{1} \prod_{j=c} \sum_{p_{j}} e^{2 \pi i p_{j} \mathbf{f}^{j} \cdot\left(\mathbf{x}-\mathbf{x}^{t}\right)},
\end{aligned}
$$

where the basis vectors belonging to $W^{c}$ and $W^{s}$ are denoted as $\mathbf{f}^{c}$ and $\mathbf{f}^{s}$, respectively. Summing up, we finally obtain

$$
\begin{aligned}
\rho(\mathbf{x})= & \prod_{j=c, s} \delta\left(\left(\mathbf{f}^{j} \cdot \mathbf{x}\right) \bmod 1\right)-\prod_{j=c} \delta\left(\left(\mathbf{f}^{j} \cdot \mathbf{x}\right) \bmod 1\right) \\
& +\frac{1}{2} \sum_{t=0}^{1} \prod_{j=c} \delta\left(\left[\mathbf{f}^{j} \cdot\left(\mathbf{x}-A^{t} \mathbf{x}^{0}\right)\right] \bmod 1\right) .
\end{aligned}
$$

This result is quite interesting and revealing. First of all, we see that the local and global properties of the system uncouple in the Fourier representation. The nontrivial structure appears as a combination of those properties, when the intersection of the "marginally stable" manifold $W^{c} \oplus W^{s}$ of the fixed point $\mathbf{k}=\mathbf{0}$, with the set $Z^{N}$, which is the reflection of the topology, contains more than one point.

Next we note that the evolution of the system is not always ergodic. In particular, if the set of basis vectors $\mathbf{f}^{c}$ is not empty (which requires nonhyperbolicity in the first place), the system has a continuum of ergodic components, whose location is determined by the initial condition. Each ergodic component is contained in a finite union of subspaces of $\mathbb{R}^{N}$, which are perpendicular to every basis vector $\mathbf{f}^{c}$, in exact agreement with the statement, proved analytically by Keller et al. (see Theorem 2 in [8]) for $a=2$ and $\varepsilon \in[0,1]$. An example of such situation is presented by the CML (14), as we will see below.

If the set of basis vectors $\mathbf{f}^{c}$ is empty, e.g., for a hyperbolic system, we find the unique invariant density [giving the natural measure $\mu(\mathbf{x})]$, in the form 


$$
\rho(\mathbf{x})=\prod_{j=s} \delta\left(\left(\mathbf{f}^{j} \cdot \mathbf{x}\right) \bmod 1\right)
$$

which reduces to Eq. (28) if the set of $\mathbf{f}^{s}$ is empty as well. Otherwise, a nonconstant invariant density results, leading to the existence of nontrivial spatiotemporal structures in the system. We should note, however, that the structure of the invariant density (41) implies that high-order correlations are expected. The widely used pair correlation functions therefore might not indicate any coherent structure, even if the latter is present.

\section{Nearest-neighbor coupling}

Up to now our conclusions have been completely general for any coupling matrix $A$ with integer elements. Let us now consider the condition (34) in more detail for our onedimensional nearest-neighbor model (14). The corresponding matrix (16) can be diagonalized by Fourier transformation in the space variables $i$, leading for periodic boundary conditions to eigenvalues

$$
\lambda_{q}=m+2 n \cos (q)
$$

and the corresponding eigenvectors

$$
\begin{aligned}
\mathbf{e}_{c}^{q} & =N^{-1}(\cos (q), \cos (2 q), \ldots, \cos (N q)), \\
\mathbf{e}_{s}^{q} & =N^{-1}(\sin (q), \sin (2 q), \ldots, \sin (N q)),
\end{aligned}
$$

where $q=2 \pi p / N$ and $p=0, \ldots, N / 2$ (for $N$ even).

Of these only a few have mutually rational components. For instance, both $\cos (q): 1$ and $\sin (2 q): \sin (q)$ are rational only if $\cos (q)$ is rational, which immediately restricts the allowed wave vectors $q=2 \pi p / N$ to a set of five values: $q^{*}=0, \pi / 3, \pi / 2,2 \pi / 3, \pi$. Each $q^{*}$ generates basis vectors $\mathbf{f}^{s}$ if $\left|\lambda_{q *}\right|<1$ and $\mathbf{f}^{c}$ if $\left|\lambda_{q *}\right|=1$ :

$$
\begin{gathered}
\mathbf{f}^{0}=(1, \ldots, 1), \\
\mathbf{f}_{1}^{\pi / 3}=(1,-1,-2,-1,1,2, \ldots, 2), \\
\mathbf{f}_{2}^{\pi / 3}=(-1,-2,-1,1,2,1, \ldots, 1), \\
\mathbf{f}_{1}^{\pi / 2}=(0,-1,0,1, \ldots, 1), \\
\mathbf{f}_{2}^{\pi / 2}=(1,0,-1,0, \ldots, 0), \\
\mathbf{f}_{1}^{2 \pi / 3}=(-1,-1,2, \ldots, 2), \\
\mathbf{f}_{2}^{2 \pi / 3}=(-1,2,-1, \ldots,-1), \\
\mathbf{f}^{\pi}=(-1,1, \ldots, 1) .
\end{gathered}
$$

Rationality of $\cos \left(q^{*}\right)$ in not an unexpected result, e.g., choosing $\cos \left(q^{*}\right)=-m / 2 n$ results in the eigenvalue $\lambda_{q^{*}}=0$, according to Eq. (42), which requires

$$
\left(\mathbf{f} \cdot \mathbf{x}^{t}\right) \bmod \quad 1=0 \quad \forall t>0,
$$

where we defined $\mathbf{f}=\kappa_{q *} \mathbf{e}^{q^{*}}$ with $\kappa_{q *}=N$ if $q^{*}=0, \pi / 2, \pi$ and $2 N$ otherwise. This in turn requires $\rho(\mathbf{x})$ $\sim \delta((\mathbf{f} \cdot \mathbf{x}) \bmod 1)$, which is seen to be the case by comparing with Eq. (41).

Similarly, the marginal eigenvalues $\lambda_{q *}= \pm 1$ also require the cosine to be rational: $\cos \left(q^{*}\right)=(-m \pm 1) / 2 n$. It can be seen that this is again consistent with the analytical result of Keller et al. (Theorem 2 of [8]). The theorem states that the marginal value of the Lyapunov exponent $\lambda_{p}=1$ for $p=N / r$ or $p=N(r-1) / r$, i.e., $q^{*}=2 \pi / r$ (or $q^{*}=2 \pi(r-1) / r$ if we allow $q$ to vary in the interval $[\pi, 2 \pi]$ ) with $r=2,3,4$, or 6 , is a sufficient condition for the existence of a continuum of ergodic components.

Since $2 \cos \left(q^{*}\right)$ can only take values $0, \pm 1$, and \pm 2 , the maximal number of basis vectors for the CML (15), (16) is $M=6$ and is achieved for $m=0, n= \pm 1$, and $N \geqslant 12$, when both the number of "central"' basis vectors $\mathbf{f}^{c}$ and the number of "stable" basis vectors $\mathbf{f}^{s}$ reach the maximum values of four and two, respectively.

From now on, however, we will restrict the discussion to the physically interesting case of ergodic dynamics. Then, there is a unique invariant density, given by Eq. (41) with $M \leqslant 2$. In order to numerically check the consistency of our results and to calculate various correlation functions, it is useful to define the one-dimensional projection of the invariant density $\rho(\mathbf{x})$ on a chosen direction $\mathbf{g}$ :

$$
\rho_{\mathbf{g}}(s)=\int_{I^{N}} \delta(s-\mathbf{g} \cdot \mathbf{x}) \rho(\mathbf{x}) d \mathbf{x},
$$

where $I^{N}$ denotes the unit $N$-dimensional cube $[0,1]^{N}$. For example, if $g_{i}=\delta_{i j}$, Eq. (46) gives the distribution of the $j$ th component of the state $\rho\left(x_{j}\right)=1$ in complete agreement with the result of [9].

If $\mathbf{g}$ coincides with one of the basis directions, i.e., $\mathbf{g}=\nu \mathbf{f}^{l}$ for some $l$, the projection

$$
\begin{aligned}
\rho_{\mathbf{g}}(s) & =\int_{I^{N}} \delta\left(s-\nu \mathbf{f}^{l} \cdot \mathbf{x}\right) \prod_{j=1}^{M} \sum_{p_{j}} \delta\left(p_{j}-\mathbf{f}^{j} \cdot \mathbf{x}\right) d \mathbf{x} \\
& =\sum_{p} D_{p} \delta(s-\nu p)
\end{aligned}
$$

becomes singular: We get a series of $\delta$ functions with an envelope

$$
D_{p}=\int_{I^{N}} \delta\left(p-\mathbf{f}^{l} \cdot \mathbf{x}\right) \prod_{j \neq l} \sum_{p_{j}} \delta\left(p_{j}-\mathbf{f}^{j} \cdot \mathbf{x}\right) d \mathbf{x} .
$$

Otherwise, the projection (46) is a continuous, nonsingular function of parameter $s$. In other words, only the projection on the directions defined by the basis vectors $\mathbf{f}^{j}$ is singular.

In particular, the eigenvector $\mathbf{e}^{q}$ defines a basis direction $\mathbf{f}^{j}$ if and only if the projection (46) on this eigenvector [we define $\rho^{q}(s)=\rho_{\mathbf{g}}(s)$ for $\left.\mathbf{g}=\mathbf{e}^{q}\right]$,

$$
\rho^{q}(s)=\int_{I^{N}} \delta\left(s-\mathbf{e}^{q} \cdot \mathbf{x}\right) \prod_{j=1}^{M} \sum_{p_{j}} \delta\left(p_{j}-\mathbf{f}^{j} \cdot \mathbf{x}\right) d \mathbf{x},
$$


One can trivially verify that the projection (49) has the average

$$
s_{q}=\int s \rho^{q}(s) d s=\frac{1}{2} \delta_{q, 0}
$$

and the dispersion given by

$$
\sigma_{q}^{2}=\int\left(s-s_{q}\right)^{2} \rho^{q}(s) d s=\frac{1}{24 N}\left(1+\delta_{q, 0}+\delta_{q, \pi}\right)
$$

for all $q \neq q^{*}$ and almost always for $q=q^{*}$. The few exceptions are all specific to short lattices: For $N=2$ and $m=2 n$ we obtain

$$
\sigma_{0}^{2}=\frac{1}{12}, \quad \sigma_{\pi}^{2}=0
$$

while $N=2$ and $m=-2 n$ yield

$$
\sigma_{0}^{2}=0, \quad \sigma_{\pi}^{2}=\frac{1}{12}
$$

$N=3$ with $m=n$ gives

$$
\sigma_{0}^{2}=\frac{1}{12}, \quad \sigma_{2 \pi / 3}^{2}=0
$$

and, finally, $N=4$ with $m=0$ and arbitrary $n$ gives

$$
\sigma_{0}^{2}=\sigma_{\pi}^{2}=\frac{1}{24}, \quad \sigma_{\pi / 2}^{2}=0
$$

It is interesting to note, however, that for $N>4$ the dispersion is given by formula (51) even for the values of parameters corresponding to nonergodic dynamics.

As expected, numerically calculating the projection $\rho^{q}(s)$ on the stable and unstable directions (43), we get a singular distribution only for $q=q^{*}$ [Fig. 2(b)], provided the respective eigenvector is stable $\left(\left|\lambda_{q}\right|<1\right)$. Otherwise a smooth Gaussian-like distribution is obtained [Fig. 2(a)].

Indeed, one can easily see that for $M=0$,

$$
\rho^{q}(s)=\int_{I^{N}} \delta\left(s-\mathbf{e}^{q} \cdot \mathbf{x}\right) d \mathbf{x}
$$

gives the probability distribution for the sum $s$ of $N$ independent weighted random variables $x_{i}$, each uniformly distributed on $[0,1]$. The weights are given by the coordinates of the eigenvector $e_{i}^{q}$. In the large length limit one can apply the central limit theorem [12], which would yield the distribution for the sum $s$, approaching a Gaussian as $N \rightarrow \infty$.

For $M>0$ Eq. (49) still gives the probability distribution for the sum of $N$ weighted random variables. However, now the variables are not independent, but correlated through the product of $M \delta$ functions (which can be simply interpreted as functional dependences of $M$ of the variables on the rest). In the large length limit this correlation can be ignored (as long as $M \ll N$ ) and the integral in Eq. (49) will still approach a Gaussian. A similar argument for the integral in Eq. (48) shows that both the continuous distribution and the envelope
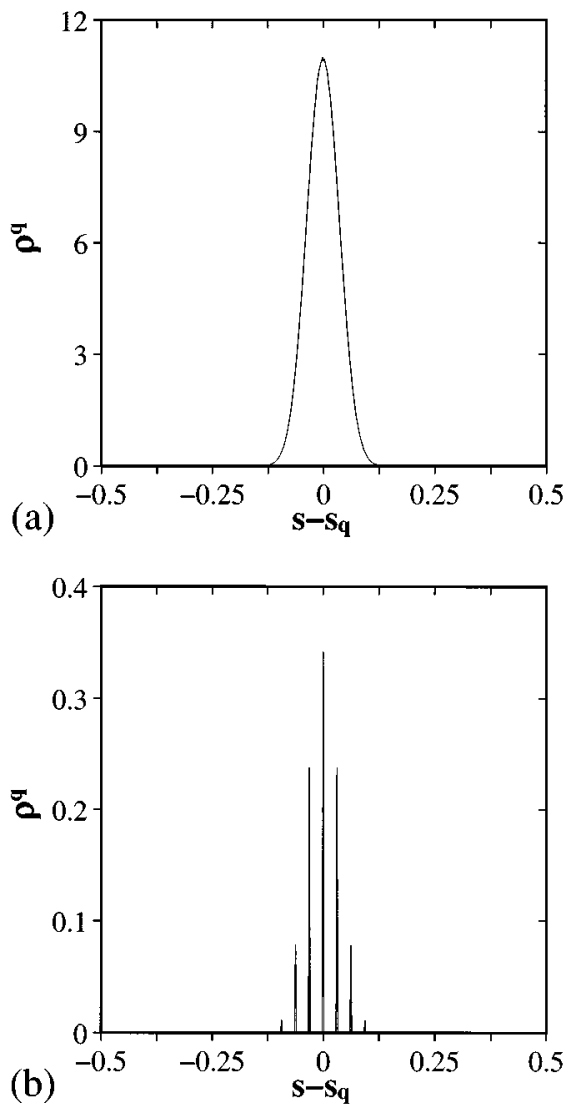

FIG. 2. Projection of the invariant density $\rho^{q}\left(s-s_{q}\right)$ for (a) $q$ $\neq q^{*}$, arbitrary $\lambda_{q}$ as well as $q=q^{*},\left|\lambda_{q}\right|>1$ and (b) $q=q^{*}$, $\left|\lambda_{q}\right|<1$. We used $N=32$.

of the singular distribution (49) become Gaussian for $N \rightarrow \infty$, independent of the number of basis vectors $\mathbf{f}^{s}$ :

$$
\rho^{q}(s) \approx \begin{cases}\frac{\nu_{q}}{\sigma_{q}} \phi\left(\frac{s-s_{q}}{\sigma_{q}}\right) \delta\left(s-\nu_{q} p\right), & \text { if } \exists j: \mathbf{e}^{q}=\nu_{q} \mathbf{f}^{j} \\ \frac{1}{\sigma_{q}} \phi\left(\frac{s-s_{q}}{\sigma_{q}}\right), & \text { otherwise, }\end{cases}
$$

where $\phi(t)=(2 \pi)^{-1 / 2} \exp \left(-t^{2} / 2\right)$ is the normalized Gaussian and $\nu_{q}=\kappa_{q}^{-1}$.

\section{Spatiotemporal correlations}

In the case of coupled map lattices the use of the standard two-point spatial correlation function is rather well motivated and is arguably the easiest way to uncover the pair correlations inherent in the system. The correlation function is trivially calculated to yield

$$
C(r)=\left\langle x_{i} x_{i+r}\right\rangle-\left\langle x_{i}\right\rangle\left\langle x_{i+r}\right\rangle=\frac{1}{12} \delta_{r, 0}
$$

for the completely expanding case with $\rho(\mathbf{x})=1$ [here \langle\rangle denotes the average taken with $\rho(\mathbf{x})]$.

If there are contracting directions, we rewrite Eq. (58) as 


$$
\begin{aligned}
C(r)= & \sum_{q}\left(\sigma_{s, q}^{2}+\sigma_{c, q}^{2}\right) e^{i q r}=\sum_{q}\left(\sigma_{s, q}^{2}+\sigma_{c, q}^{2}-\frac{1}{12 N}\right) e^{i q r} \\
& +\frac{1}{12} \delta_{r, 0}
\end{aligned}
$$

where $\sigma_{s, q}=\sigma_{c, q}=\sigma_{q}$ for all $q$ except $\sigma_{s, 0}=\sigma_{s, \pi}=0$. Since $\sigma_{q}^{2}=\left(1+\delta_{q, 0}+\delta_{q, \pi}\right) / 24 N$ for all $q \neq q^{*}$,

$$
\begin{aligned}
C(r)= & \frac{1}{12} \delta_{r, 0}+\left(\sigma_{0}^{2}-\frac{1}{12 N}\right)+\left(\sigma_{\pi}^{2}-\frac{1}{12 N}\right)(-1)^{r} \\
& +4 \sum_{q=\pi / 3, \pi / 2,2 \pi / 3}\left(\sigma_{q}^{2}-\frac{1}{24 N}\right) \cos (q r) .
\end{aligned}
$$

This reduces to a $\delta$ correlation [which coincides with the result (58) obtained for $\rho(\mathbf{x})=1]$ in all but a few special cases described above, when $\sigma_{q}^{2} \neq\left(1+\delta_{q, 0}+\delta_{q, \pi}\right) / 24 N$. For instance, choosing $m=0$ yields, for $N=4$, the invariant density

$$
\rho(\mathbf{x})=\delta\left(x_{1}-x_{3}\right) \delta\left(x_{2}-x_{4}\right)
$$

and according to Eqs. (55) and (60)

$$
C(r)=\frac{1}{24}+\frac{(-1)^{r}}{24}= \begin{cases}\frac{1}{12} & \text { if } r=0,2 \\ 0 & \text { if } r=1,3 .\end{cases}
$$

Since the invariant density, although being nontrivial, does not tell us much about the spatiotemporal structures in the system, next we introduce a spatiotemporal correlation function $G_{i}(r, t)$, which is a straightforward generalization of the time correlation function (11):

$$
G_{i}(r, t)=\int d \mathbf{x}^{0} \rho\left(\mathbf{x}^{0}\right) e^{2 \pi i\left(x_{i}^{0}-x_{i+r}^{t}\right)} .
$$

By expanding $\rho(\mathbf{x})$ into Fourier series we obtain, in analogy to Eq. (25),

$$
G_{i}(r, t)=\sum_{\mathbf{k}} \hat{\rho}(\mathbf{k}) \prod_{j=1}^{N} \delta\left(k_{j}-A_{i+r, j}^{t}+\delta_{i, j}\right) .
$$

Since only the nonvanishing Fourier components $\hat{\rho}\left(\mathbf{k}^{*}\right)=1$ (where $\mathbf{k}^{*}=\Sigma_{l} n_{l} \mathbf{f}^{l}$ ) of the invariant density (41) contribute, Eq. (64) reduces to

$$
G_{i}(r, t)=\sum_{n_{1}, \ldots, n_{M}} \prod_{j=1}^{N} \delta\left(\sum_{l=1}^{M} n_{l} f_{j}^{l}-A_{i+r, j}^{t}+\delta_{i, j}\right) .
$$

In a translationally invariant system $G_{i}(r, t)$ does not depend on $i$, so we drop the index and fix $i$ (set $i=1$ to be specific).

It can be easily verified that the correlation (63) is short ranged in both space and time. First we note that it vanishes if the vector $\mathbf{k}_{r}^{t}$ with components $k_{j}=A_{1+r, j}^{t}-\delta_{1, j}$ does not lie on the stable manifold $W^{s}$. According to Eq. (29),

$$
A_{1+r, j}^{t}=\lambda_{1}^{t}\left[e_{1+r}^{1} e_{j}^{1}+\left(\frac{\lambda_{2}}{\lambda_{1}}\right)^{t} e_{1+r}^{2} e_{j}^{2}+\cdots\right],
$$

where $\lambda_{1}$ is the largest and $\lambda_{2}$ the next largest eigenvalue and $\mathbf{e}^{1}$ and $\mathbf{e}^{2}$ are the respective eigenvectors. For increasing $t$ the vector $\mathbf{k}_{r}^{t}$ asymptotically approaches the direction defined by $\mathbf{e}^{1}$ and therefore cannot lie on the stable manifold for $t \geqslant \tau$, where $\tau$ is some finite (and typically small) integer.

On the other hand, for $t=0$ we have

$$
G(r, 0)=\sum_{n_{1}, \ldots, n_{M}} \prod_{j=1}^{N} \delta\left(\sum_{l=1}^{M} n_{l} f_{j}^{l}-\delta_{1+r, j}+\delta_{1, j}\right)
$$

and one can easily see that equal-time spatial correlations vanish for sufficiently large lattices. Indeed, in the translationally invariant case any possible basis vectors (44) are periodic in space with periods $1,2,3,4$, or 6 and any linear combination of these will also be periodic with period of at most 12. Since the vector with components $k_{j}=\delta_{1+r, j}-\delta_{1, j}$ is not periodic for $r \neq 0$, the maximal size of the system with nontrivial correlation is limited to $N=12$. Again, considering the CML (14) with $m=0$ and $N=4$ as an example, we obtain $\mathbf{f}^{1}=(0,-1,0,1)$ and $\mathbf{f}^{2}=(1,0,-1,0)$ as the basis vectors and consequently

$$
G(r, 0)=\left\{\begin{array}{lll}
1 & \text { if } & r=0,2 \\
0 & \text { if } & r=1,3,
\end{array}\right.
$$

i.e., we retrieve the result (62).

The results obtained above for two different two-point correlation functions should serve as a warning for using low-order correlations as an indicator of the existence of coherent structures. Although our model is admittedly rather special, we might suggest that certain types of coherent structures will generically only transpire through the highorder correlations such that the order is comparable to the number of degrees of freedom of the dynamical system considered.

\section{DISCUSSION}

To summarize, we have shown that the solution for the dynamical behavior of a lattice of Bernoulli maps, which are coupled by a matrix $A$ with integer coefficients, can be given in the closed form as $\mathbf{x}^{t}=\left(A^{t} \mathbf{x}^{0}\right) \bmod 1$, i.e., the dynamical behavior of the coupled map system can be described by the repeated action of a linear map $A^{t} \mathbf{x}^{0}$ on variables that are confined to an $N$-torus. This picture explains that the essentials of the dynamical behavior are dictated by the eigenvalues and eigenvectors of $A$.

We have also calculated the invariant density and a number of correlation functions, and it is instructive to compare our results with the general results obtained by Bunimovich and Sinai [5], who proved that for sufficiently small coupling (in our case determined by the parameter $\varepsilon$ ) certain expanding coupled map systems with finite-range coupling possess an invariant measure $\mu(\mathbf{x})$, whose finite-dimensional distributions are absolutely continuous. Furthermore, it was proved that the time and space correlation functions decay exponentially (not slower than exponentially, to be exact).

Indeed, small coupling in our model means that there are no contracting directions and as a result we have a completely expanding system with the unique invariant measure that has constant density $\rho(\mathbf{x})=1$. For larger coupling, the 
invariant measure might still exist, but might not be absolutely continuous due to the fact that large coupling often causes the appearance of contracting directions even if the isolated local maps $f(x)$ are expanding. For certain values of control parameters, however, the invariant measure disappears, giving way to the continuum of ergodic components, selected by the choice of initial conditions. Although our model suggests that the existence of the central manifold is the crucial ingredient in the violation of ergodicity, it remains to be seen whether it constitutes the necessary condition in general.

We find that the invariant density $\rho(\mathbf{x})$ of the system (15) displays Fourier coefficients that are different from zero, i.e., $\rho(\mathbf{x})$ is nonconstant, whenever the stable manifold of the zero wave vector contains a nonempty basis of directions $\mathbf{f}^{j}$ with mutually rational components, generating an infinite asymptotically contracting set of wave vectors. For nearestneighbor couplings in a one-dimensional lattice [given by the matrix (16)] the maximal number of such basis vectors is six (two for ergodic dynamics).

The standard spatial correlation function $C(r)$ for the model with nearest-neighbor couplings is given by $C(r)=(1 / 12) \delta_{r, 0}$ for almost any combination of control parameters. A few special cases exist, however, for sufficiently small lattices, where the spatial correlations are different. We can interpret this result by noting that the order of the correlation function, i.e., 2, is indeed comparable to the length of the lattice with nontrivial correlations, i.e., $N \leqslant 4$. Nevertheless, $C(r)$ always vanishes at sufficiently large distances, which is consistent with [5].

Furthermore, it is rather interesting to note that both the Lyapunov spectrum and (for sufficiently large lattices) the spatial correlation function not only can be calculated exactly, but do not depend on the initial conditions for arbitrary values of system parameters, even when the dynamics of the system is not ergodic. Such dynamical invariants, although not universal, should be very helpful in describing nonergodic dynamical systems.

The invariant density and the spatial correlation function of the model considered here display little structure compared to the Lyapunov spectrum, which is, for the nearestneighbor coupling, given by $\Lambda_{q}=\ln |m+2 n \cos (q)|$. This result shows that the time-averaged spatial behavior is not simply a straightforward reflection of the Lyapunov spectrum (see related works listed in [13]).

We have also calculated the measurable spatiotemporal correlation function $G(r, t)$ for the translationally invariant model and shown that it too is short ranged in both space and time for arbitrary coupling matrix $A$. This suggests that space and time correlations can decay exponentially or faster even in the systems with global coupling.

Summing up the main points, we might suggest that there exists a class of spatiotemporally chaotic systems that display no coherent structures in certain regions of parameter space. However, even if the coherent structure is present, the spatiotemporal correlations might decay subexponentially or even show $\delta$ correlations. In the latter case higher-order correlation functions are necessary to discover the coherent structure.

Furthermore, we can suggest that the dynamics of certain spatiotemporally chaotic systems could lose the ergodicity property on some hypersurface of the parameter space. When this happens, the Lyapunov spectrum and finite-dimensional spatiotemporal correlation functions could provide us with initial-condition-independent information about the system dynamics.

Let us finally point out several directions of further research. One open problem is the extension of our results to higher dimensions and to couplings that have a longer range. In the one-dimensional case the eigenvectors remain also valid for longer-ranged couplings; only the eigenvalues change. This means that a model with a long but finite range will have no more structure in the invariant density than the short-ranged model. This is of course a peculiarity of the Bernoulli shift map, but should again be taken as a warning for making conclusions from the spatial range of the coupling onto the observable spatial patterns.

Although our solution for the dynamics and the correlation functions does hold for general dimensions, it would be interesting to see what the restrictions on the wave vectors that generate the basis of the invariant density look like in two and three dimensions. The study of the model with noninteger coupling and expansion rates using a sort of perturbation technique around an exact solution also seems promising.

Finally, one could investigate the dynamical behavior of a system, whose time dependence is given a priori by Eq. (20) for matrices $A$ with noninteger elements. By doing so one will lose the property of the original map that relation (17) holds step by step, although the trajectories generated by Eq. (20) are well defined.

\section{ACKNOWLEDGMENTS}

The authors thank M. C. Cross for the careful reading of the manuscript. H.G.S. thanks C. Koch for the kind hospitality extended to him at Caltech and the Volkswagen Foundation for financial support. This research has also been partially supported by the NSF through Grant No. DMR9013984.
[1] U. Frisch, Turbulence (Cambridge University Press, New York, 1995).

[2] H. G. Schuster, Deterministic Chaos, 3rd ed. (VCH, New York, 1995); J. L. McCauley, Chaos, Dynamics and Fractals (Cambridge University Press, New York, 1993).

[3] K. Kaneko, Prog. Theor. Phys. 72, 480 (1984); I. Waller and K. Kapral, Phys. Rev. A 30, 2047 (1984); J. Crutchfield, Physica D 10, 229 (1984).
[4] K. Kaneko, Chaos 2, 279 (1992); Coupled Map Lattices, edited by K. Kaneko (World Scientific, Singapore, 1992).

[5] L. A. Bunimovich and Ya. G. Sinai, Nonlinearity 1, 491 (1988); in Theory and Applications of Coupled Map Lattices, edited by K. Kaneko (Wiley, New York, 1993).

[6] A. Politi and G. R. Puccioni, Physica D 58, 384 (1992); K. Kaneko, Phys. Lett. A 139, 47 (1989).

[7] V. I. Arnold and A. Avez, Ergodic Problems of Classical Me- 
chanics (Benjamin, New York, 1968).

[8] G. Keller, M. Kunzle, and T. Nowicki, Physica D 59, 39 (1992), and references therein.

[9] C. Diks, F. Takens, and J. DeGoede, Physica D 104, 269 (1997).

[10] Similar correlation functions are used for the $X Y$ model. J. V. Jose, L. P. Kadanoff, S. Kirkpatrick, and D. R. Nelson, Phys. Rev. B 16, 1217 (1977).

[11] J.-P. Eckmann and D. Ruelle, Rev. Mod. Phys. 57, 617 (1985).
[12] W. Feller, An Introduction to Probability Theory and its Applications, 3rd ed. (Wiley, New York, 1970).

[13] H. Daido, Prog. Theor. Phys. 72, 853 (1984); F. Kaspar and H. G. Schuster, Phys. Lett. A 113, 451 (1986); T. Bohr and O. B. Christensen, Phys. Rev. Lett. 63, 2161 (1989); C. Nicolis, G. Nicolis, and Q. Wang, Int. J. Bifurcations Chaos 2, 263 (1992); W. Yang, E. J. Ding, and M. Ding, Phys. Rev. Lett. 76, 1808 (1996). 